\title{
Metastatic Malignant Neoplasm in the Adrenal Gland
}

National Cancer Institute

\section{Source}

National Cancer Institute. Metastatic Malignant Neoplasm in the Adrenal Gland. NCI

Thesaurus. Code C8610.

A malignant tumor that has spread to the adrenal gland from an adjacent or distant anatomic site. The majority of cases are metastatic carcinomas, and less frequently lymphomas. 